\title{
The Nature of Ultraluminous Infrared Galaxies
}

\author{
Eckhard Sturm \\ Max-Planck Institut für extraterrestrische Physik, Postfach 1312, 85741 \\ Garching, Germany
}

\begin{abstract}
.
The Infrared Space Observatory (ISO) has for the first time made possible mid- and far-infrared spectroscopic studies of nearby and moderate redshift, dusty ultraluminous infrared galaxies (ULIRGs). We discuss recent SWS, LWS, ISOPHOT-S and ISOCAM (CVF) results on ULIRGs, addressing the following topics: What powers ULIRGs? What is the AGN-starburst connection? How do ULIRGs evolve? What is the connection of the local ULIRG population to the recently discovered population of mid-IR to submillimeter sources?
\end{abstract}

\section{Introduction}

ULIRGs are the dominant population of extragalactic objects for $\mathrm{L}>10^{12} \mathrm{~L}_{\odot}$, with space densities similar to or exceeding that of quasars at comparable bolometric luminosity. They are predominantly galactic mergers (Figure 1). ULIRGs are heavily obscured by dust, and more than $90 \%$ of the bolometric luminosity is re-processed by this dust and re-emitted in the infrared. The high extinction makes it impossible to study the central regions of ULIRGs at UV, optical or even near-infrared wavelengths, which is why the source of the enormous luminosity (powerful starbursts or AGNs) has been a subject of much debate during the last 15 years (see, for instance, the Proceedings of the Ringberg Conference "Ultraluminous Galaxies: Monsters or Babies?", Astrophysics and Space Science, Vol. 266, Nos. 1-2, 1999).

While, locally, these infrared bright galaxies contribute only $1 \%$ to the infrared radiation field, and only $0.3 \%$ to the bolometric radiation field (Sanders \& Mirabel 1996; Heckman 1999), they become much more important at higher redshifts because of the higher frequency of galaxy collisions and the larger fraction of unprocessed gas. Collisions and mergers of galaxies play a critical role in the evolution of galaxies (Sanders \& Mirabel 1996; Genzel, Lutz, \& Tacconi 1998a). Hierarchical merging of smaller sub-units into bigger ones was likely a key process in the formation of galaxies. In fact, many authors have argued that the role of mergers becomes increasingly important with increasing redshift (Zepf \& Koo 1989; Carlberg, Pritchet, \& Infante 1994; Neuschaefer et al. 1997), and characterize the merger rate as $(1+z)^{m}$, where $m$ is between 1 and 4 . Hence, ULIRGs are excellent local templates for the processes by which galaxies were built and by which the intergalactic medium was heated and chemically enriched. There 

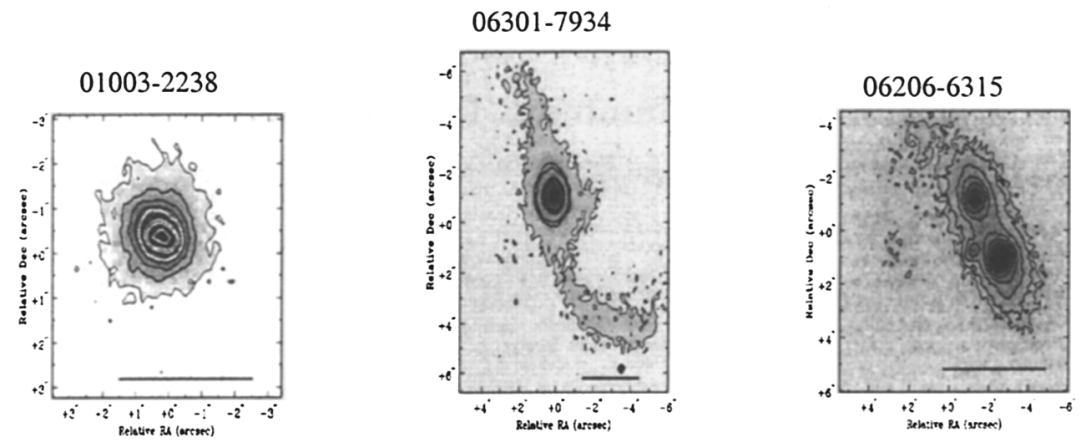

Figure 1. Three examples of near-infrared ULIRG images, representing three different stages of merger evolution (from Rigopoulou et al. 1999).

exists very likely an evolutionary link between ULIRGs, starbursts, QSOs, and ellipticals (see section 4.2).

\section{The Importance of ULIRGs for the Extragalactic IR Background}

Ground-based and HST deep rest-frame UV surveys and spectroscopic followup indicate that the integrated star formation rate in the Universe increases with increasing redshift up to $z=1$ to 2 , and then possibly decreases again at higher redshift (Steidel et al. 1999; Madau et al. 1996; Pettini et al. 1998). A significant new element in the picture of high- $z$ star formation has emerged with the COBE detection of an extragalactic sub-millimeter background (Puget et al. 1996). This diffuse background has an integrated intensity that is comparable to or larger than that of the integrated UV/optical light of galaxies (Dwek et al. 1998; Hauser et al. 1998; Lagache et al. 1999). The implication is that there likely exists a very significant contribution of dust obscured star formation at high redshifts that has been missed in the UV surveys. Consequently, large extinction corrections to the optical luminosity function have to be accounted for. In deep mid-infrared surveys, the Infrared Space Observatory (ISO) has been able to provide for the first time a glimpse of the infrared emission of galaxies at $z \geq 0.5$ and to resolve some of the galaxies that are responsible for the far-infrared and sub-millimeter background (see Genzel \& Cesarsky 2000 and references therein). At the same time, SCUBA surveys (Holland et al. 1999; Barger et al. 1999 and references therein) have uncovered a substantial population of dusty galaxies with properties similar to those expected for distant counterparts of local ULIRGs, e.g., similar morphologies, luminosities, and spectral energy distributions (SEDs). The latter fact is illustrated in Figure 2, which compares the SED of one of the SCUBA sources to the SED of a local ULIRG, redshifted to $z \sim 4$. In summary, dusty infrared bright galaxies seem to have been responsible for a significant fraction of the high-mass star formation and associated heavy element enrichment in the history of the universe. 


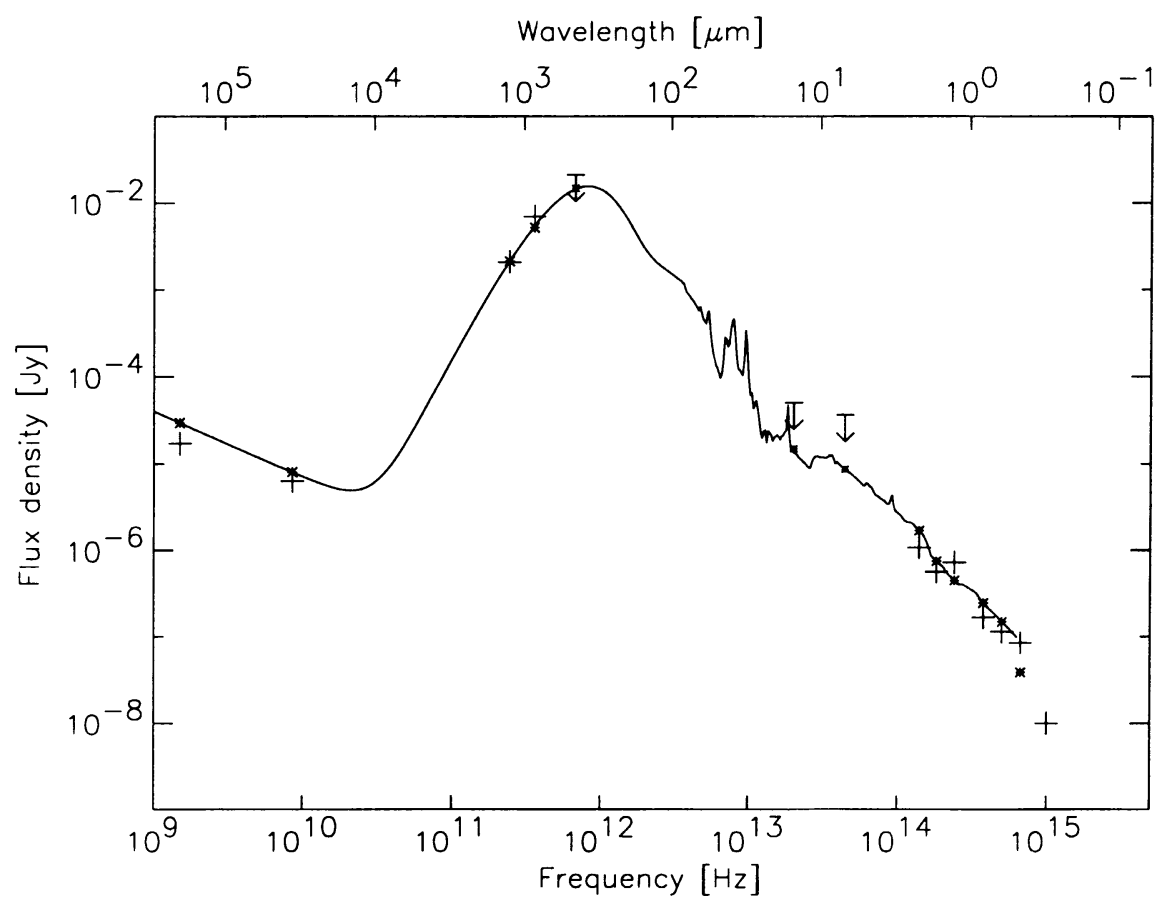

Figure 2. Spectral energy distribution of the SCUBA source HDF 850.1 (Hughes et al. 1998, Downes et al. 1999), overplotted with a ULIRG SED redshifted to $z \approx 4$ (D. Lutz, private communication).

Recent x-ray observations with ROSAT, ASCA and BeppoSax have also added substantially to our picture of ULIRGs. The discovery that some fraction of the X-ray background appears to be produced by a population of heavily obscured AGNs (e.g., Fabian \& Barcons 1992; Almaini et al. 1998), objects which have been largely missed in optical surveys due to extremely heavy obscuration, has clearly further increased the importance of studies of infrared-selected galaxies. A large contribution of hidden AGNs to the far-IR background would complicate the deduction of the star formation history of the universe from galaxy luminosity functions.

\section{Mid-Infrared Diagnostics and Results from ISO}

The 1995-1998 ISO mission provided detailed mid- and far-infrared spectroscopic and spectrophotometric observations of galaxies. With such observations, quantitative information about the physical characteristics of the emitting gas in obscured galaxies (starbursts, ULIRGs, AGNs) can be obtained from line ratios. The ratios from different ionic states are powerful probes of the hardness of the radiation field and the ionization parameter (e.g., [NeII], [NeIII], [Ne V], $[\mathrm{Ne} \mathrm{VI}]$ ) and also reflect the local gas density (e.g., [S III], [Ne III], [Ne V]). Hydro- 
gen recombination lines and line ratios like [S III] $33 \mu \mathrm{m} / 18 \mu \mathrm{m}$ trace the mid-IR extinction. Models and diagnostic diagrams have been developed that use line ratios, abundances, bolometric and ionizing luminosities, and other parameters that can be deduced from mid-IR spectroscopy to understand the nature of and the physical processes occuring within galactic nuclei. The ISO-SWS spectrum of the Circinus galaxy (Figure 3, Moorwood et al. 1996; Sturm et al. 2000) typifies the key constituents of mid-infrared spectra of galaxies:

(a) A dust continuum rising towards longer wavelengths,

(b) Emission features from transiently heated aromatic material ("PAH features"),

(c) A rich hydrogen recombination line and fine structure line spectrum from material ionized by young stars and/or an AGN,

(d) Tracers from PDRs, shocks, X-ray excited gas, such as rotational $\mathrm{H}_{2}$ lines from warm molecular material and [FeII] from partially ionized zones, and

(e) Absorption features, the strongest ones at 9.6 and $18 \mu \mathrm{m}$ being due to silicate dust.

An important application of ISO has been to distinguish between star formation and AGN activity in obscured environments, in particular the dust-rich ultraluminous infrared galaxies (ULIRGs). These analyses make use of two simple diagnostics (Figure 4). Firstly, emission of high excitation fine structure lines is strong in the narrow line region (NLR) of AGN but very weak in starbursts. Secondly, the PAH features are ubiquitous in normal galaxies and starbursts but absent near an AGN, as shown by spatially resolved ISOCAM observations (Laurent et al. 1999, Moorwood et al. 1996).

\section{Results}

\subsection{Nature of ULIRGs}

Genzel et al. (1998b) have applied these diagnostics to a sample of 15 ULIRGs observed at high resolution with ISO-SWS and at low resolution with ISOPHOT$\mathrm{S}$, and find that most of them are powered predominantly by starbursts (Figure 5). Lutz et al. (1998), Rigopoulou et al. (1999) and Tran et al. (2001) have extended this work to larger samples, using the PAH diagnostic to reach fainter sources. Their results show that there is a transition towards AGN dominance at the highest luminosities (Figure 6). However, some starburst-dominated systems are found up to luminosities of at least $5 \times 10^{12} \mathrm{~L}_{\odot}$. Since there is ample evidence for co-existence of AGN and starbursts in at least some ULIRGs it is necessary to estimate quantitatively how much starburst and AGN contribute to the bolometric luminosity of the system. For the starburst component, quantitative estimates have been attempted by Genzel et al. (1998b), who inferred the starburst ionizing luminosity from the emission lines, and by Rigopoulou et al. (1999), who used the PAH luminosity. Both papers conclude that in most objects the star formation actually detected is sufficient to power at least half of the total luminosity, leaving little room for other power sources. This conclusion is further supported by Sturm et al. (2000) and Tran et al. (2001), who have developed a method to quantitatively decompose mid-IR spectra into the different 


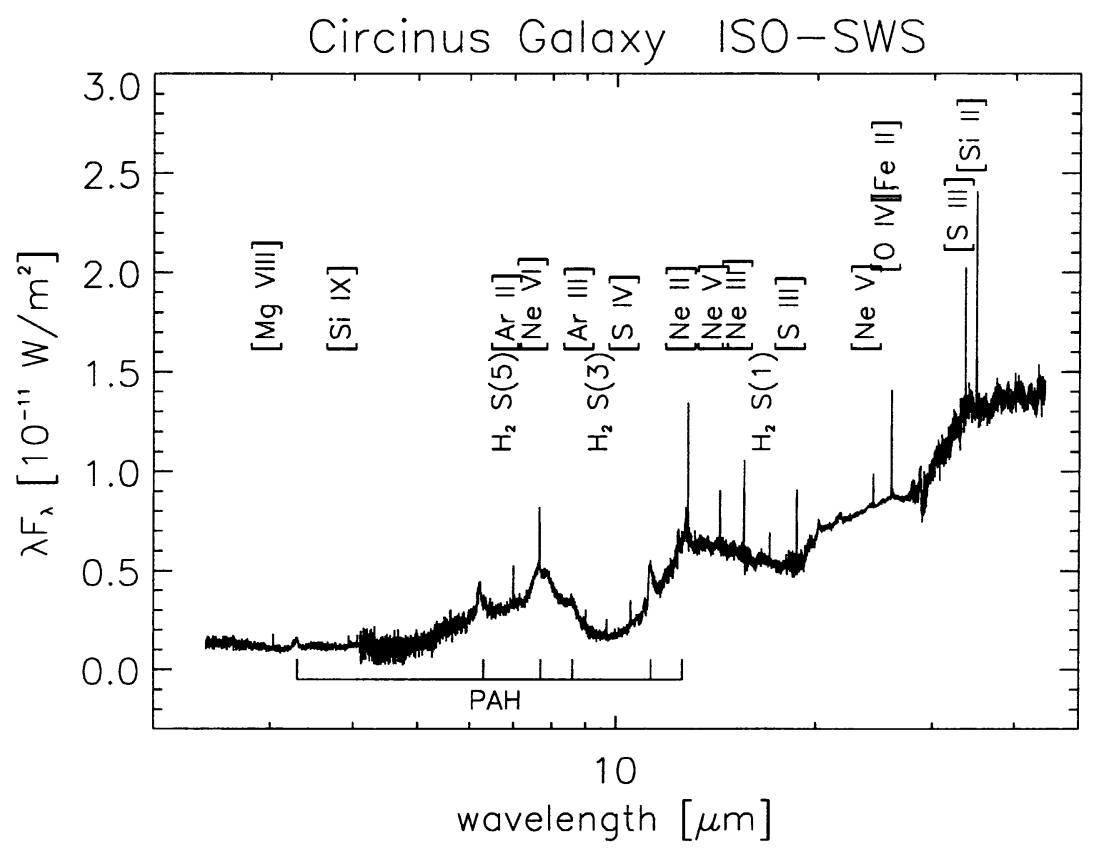

Figure 3. 2.4-45 $\mu \mathrm{m}$ ISO-SWS spectrum of the Circinus Galaxy. This composite object shows indicators of both AGN and starburst activity.

Starburst NGC 3256
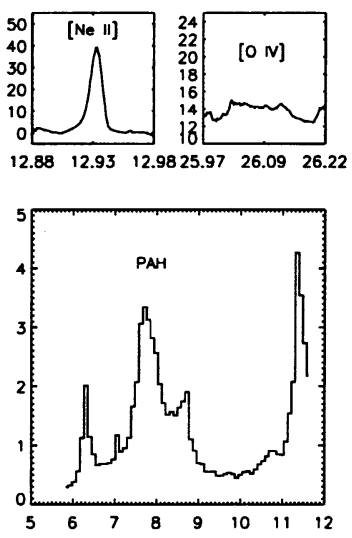

ULIRG

Arp 220
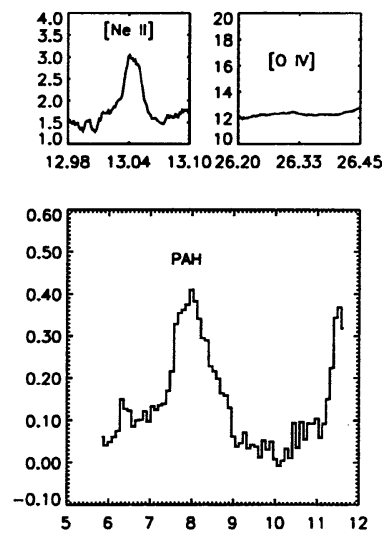

AGN

NGC 4151
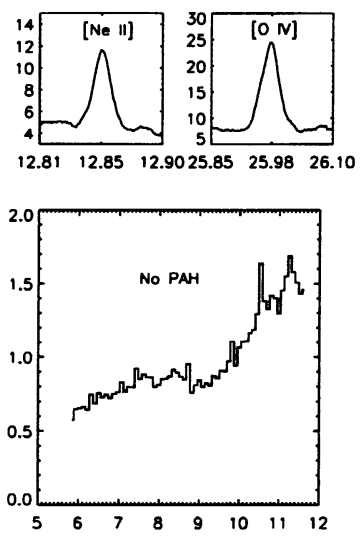

Figure 4. The two ISO diagnostics of AGN and starburst activity: a large ratio of high (e.g., [OIV]) versus low excitation (e.g. [NeII]) fine structure lines indicates an AGN, while strong "PAH" emission features trace star formation activity. In both aspects, the ULIRG Arp 220 is more similar to a starburst than to an AGN. 

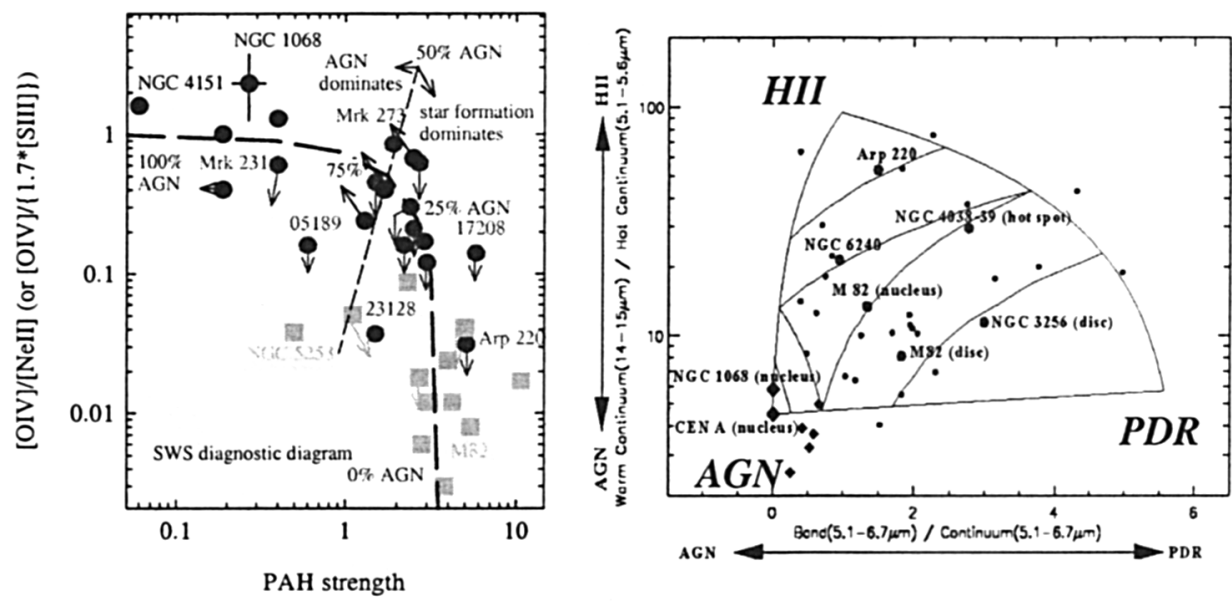

Figure 5. Left: ISO diagnostic diagram combining the fine structure line and the PAH diagnostic for about 15 ULIRGs and comparison templates (Genzel et al. 1998b). Most ULIRGs appear predominantly starburst-powered. In many cases, the quality of the [O IV] upper limits is good enough to indicate starburst dominance, but not sufficient to quantify a possible minor AGN contribution. Right: ISOCAM diagnostic diagram (Laurent et al. 2000) introducing the additional element of continuum slope as an AGN vs. intense star formation ("HII") discriminant.

contributions from starforming regions and AGNs. The composite spectra are fitted with a mixture of templates for PDRs, HII regions, an AGN continuum, and extinction. Examples of such quantitative fits are shown in Figures 7 and 8.

Lutz, Veilleux, \& Genzel (1999) have compared ISO classifications (starburst or AGN) with classifications from optical spectroscopy. The agreement between mid-infrared and optical classification is excellent, if optical LINER spectra are assigned to the starburst group. LINER spectra in infrared-selected galaxies, rather than being an expression of the AGN phenomenon, are probably due to shocks that are related to galactic superwinds. The comparison also suggests that AGN activity, once triggered, quickly breaks the obscuring screen at least in certain directions, thus becoming detectable over a wide wavelength range.

\subsection{Evolution}

One of the most fundamental problems in extragalactic astronomy is the origin of the quasar activity in galaxies and its possible link to galaxy formation. The recent discovery that all massive ellipticals appear to contain massive black holes (e.g., Joseph 1999 and references therein) suggests a direct link between the formation of spheroids and the growth of black holes at the centers of galaxies. Several models have been proposed to explain these results. In one scenario, a global interaction mechanism is presumed to link ULIRGs with starbursts, 


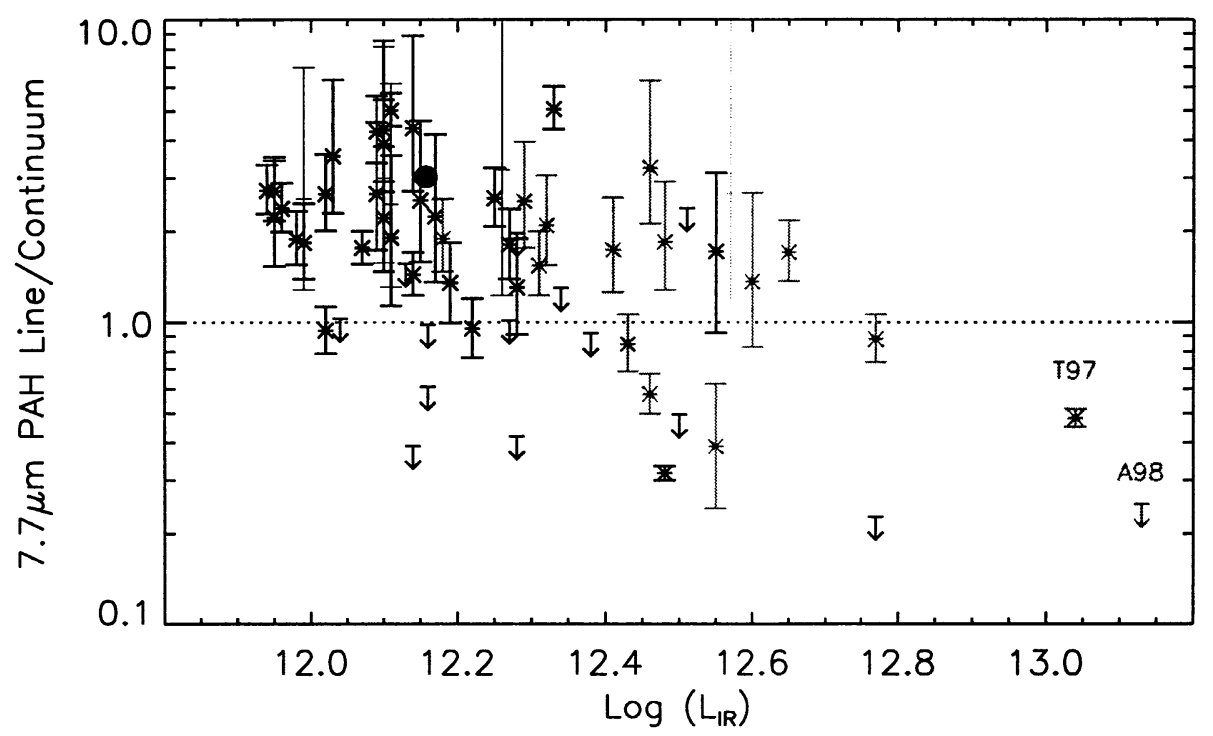

Figure 6. Line to continuum ratio of the $7.7 \mu \mathrm{m}$ PAH feature as a function of the IR luminosity (black: ISOPHOT-S data, grey: ISOCAM-CVF data). Points above 1.0 are starburst dominated (Tran et al. 2001).

QSOs, and ellipticals (e.g., Sanders et al. 1988; Kormendy \& Sanders 1992). According to this scenario, interactions and merging of the ULIRG parent galaxies cause gas to be transported to the inner parts of the galaxies. This central gas concentration triggers powerful starburst activity. As the merger proceeds, the starburst activity subsides and the luminosity increases rapidly and dominates the bolometric output during the ULIRG phase. Eventually, all the obscuring dust is shed and the ULIRG develops into a normal QSO. When the starburst and AGN activity finally have ceased and the gas is either used up or expelled in a wind, the final state of a ULIRG merger may be a large elliptical galaxy with a massive quiescent black hole at its center (e.g., Kormendy \& Sanders 1992), as indicated, e.g, by the $\mathrm{r}^{1 / 4}$-law brightness profiles of the outer parts of advanced mergers (Joseph \& Wright 1985; Surace \& Sanders 1999; Veilleux et al. in prep.).

In this scenario one would expect that more advanced mergers are more AGN-like. Current results on a limited set of ULIRGs by Rigopoulou et al. 1999 do not confirm such a trend. Using the projected separation of the double nuclei to determine the evolutionary state of these ULIRG mergers they find AGN- or starburst-dominated ULIRGs at all nuclear separations (Figure 9). This suggests that the dominance of AGN or starburst in ULIRGs may depend on local and short-term conditions (e.g., compression of the circumnuclear interstellar medium as a function of gas content and galaxy structure, local accretion rate onto the central black hole, etc.) in addition to the global state of the merger. Still, several lines of evidence suggest that warm ULIRGs are in- 


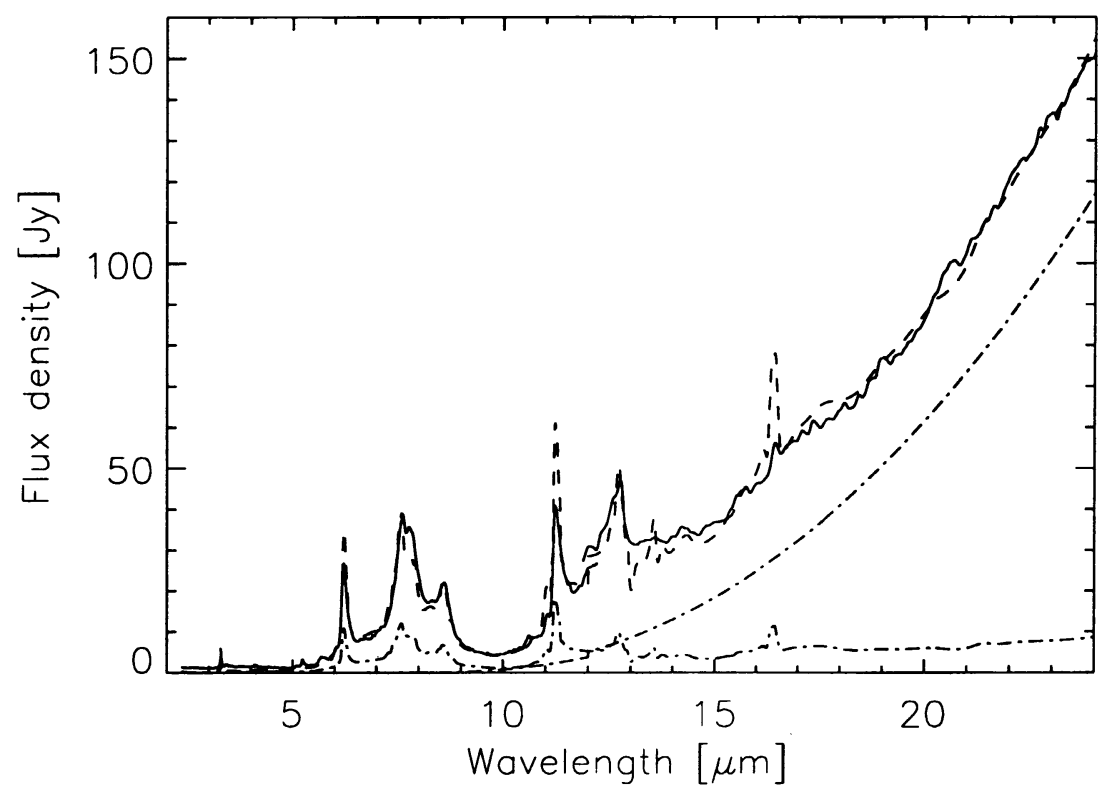

Figure 7. A simple fit to M82 (full) by a scaled combination of the reflection nebula NGC7023 (a pure PDR/PAH spectrum without strong continuum and extinction) and a power-law continuum (representing a hot component from small dust grains), dash-dotted. No extinction has been applied. From Sturm et al. (2000).

deed more advanced, transition objects and that (radio quiet) QSOs correspond to the final state of this sequence (e.g., Surace \& Sanders 1999). The final product may be a relaxed elliptical (Kormendy and Sanders 1992), but this proposal still needs to be tested. For instance, young proto-ellipticals/post-mergers might still have a significant amount of dust, which could hide signs of (weak) accreting black holes in optical studies. Such tracers could be found in the future with sensitive mid-infrared spectroscopy (e.g., SIRTF-IRS). In addition, their position in the diagnostic diagrams will show whether there are connecting trends between ULIRGs, QSOs and (proto-)ellipticals, possibly along a merger sequence. Another strong link between ULIRGs and ellipticals, independent of a merger hypothesis, can be made by noting that the metallicities - particularly the $\mathrm{H} \alpha$-to-iron ratios - of ellipticals indicate that they were formed via strong starbursts.

\section{Open problems}

ISO has opened a new window on galaxies, and new diagnostics have been demonstrated for discriminating starburst and AGN activity in obscured galaxies 
and for in-depth understanding of the "templates" involved. However, due to the moderate signal-to-noise of the individual data and the modest sample sizes achieved, ISO has left many problems unsolved and has posed new questions.

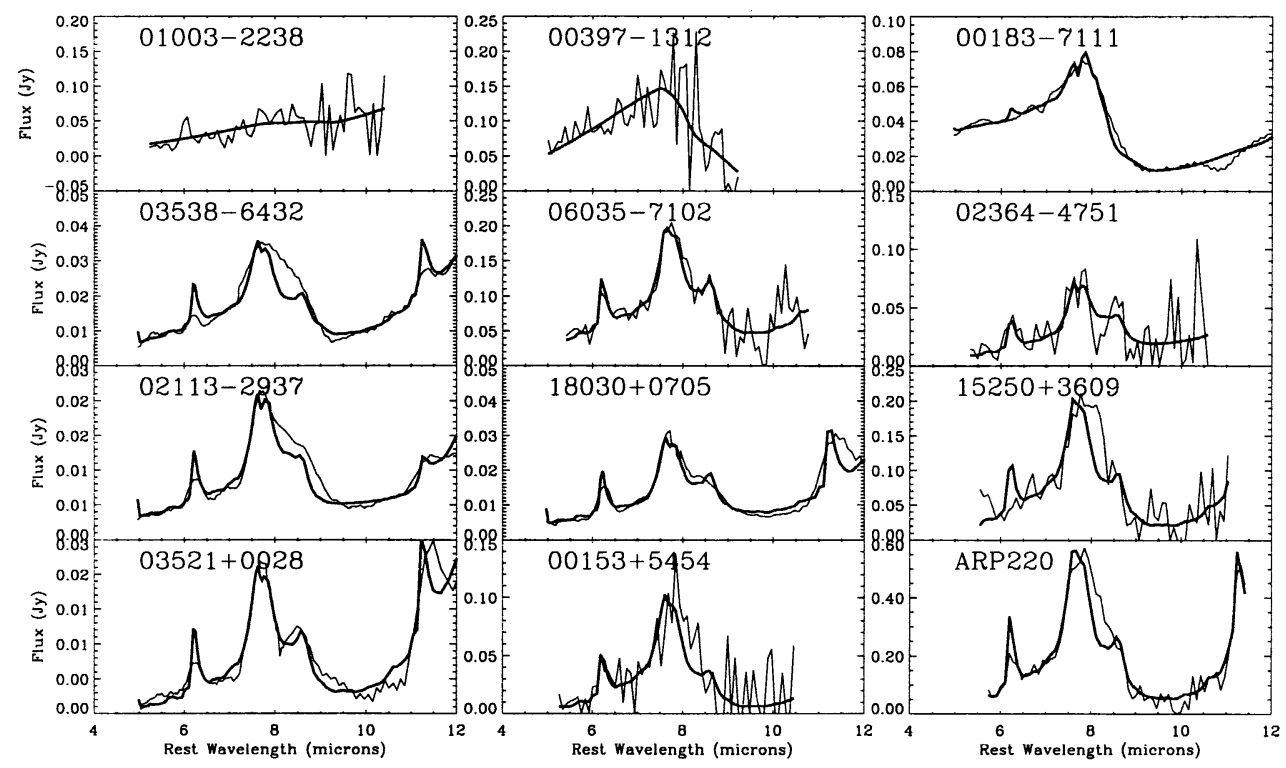

Figure 8. Examples of model fits to (CAM-CVF) ULIRG spectra. The model applies a starburst/PAH template (M82) plus a continuum component (power law), representing a hot dust continuum either from a strong starburst radiation field (additional PDR) or from an AGN. The extinctions to the starburst and AGN components are treated independently from each other. These kinds of fits permit an improved quantification of the relative contributions of star formation and AGN activity to the mid-IR emission of ULIRGs. From Tran et al. (2001).

For instance, for most of the ULIRGs observed with ISO, the classifications in the x-ray (ASCA), optical and in the mid-infrared agree quite well (Genzel \& Cesarsky 2000; Lutz, Veilleux, \& Genzel 1999), i.e. those (U)LIRGs known to harbor an active nucleus in the x-ray or optical also show the typical high excitation lines from the narrow line region in the infrared, and/or have weak PAH features. However, some (U)LIRGs, like NGC 4945 are particularly puzzling. Their mid-infrared spectra present strong PAH features which indicate a low UV radiation field (Spoon et al. 2000). The fine structure lines of high ionization potential are weak compared to AGNs. All those mid-infrared indicators are similar to those observed in starburst galaxies and indicate that most, if not all, of the bolometric luminosity can be explained by starbursts alone. Nevertheless, hard x-ray observations have shown that these galaxies harbor a Compton-thick AGN which could completely dominate the bolometric energy output (Iwasawa et al. 1993; Iwasawa \& Comastri 1998; Guainazzi et al. 2000). 


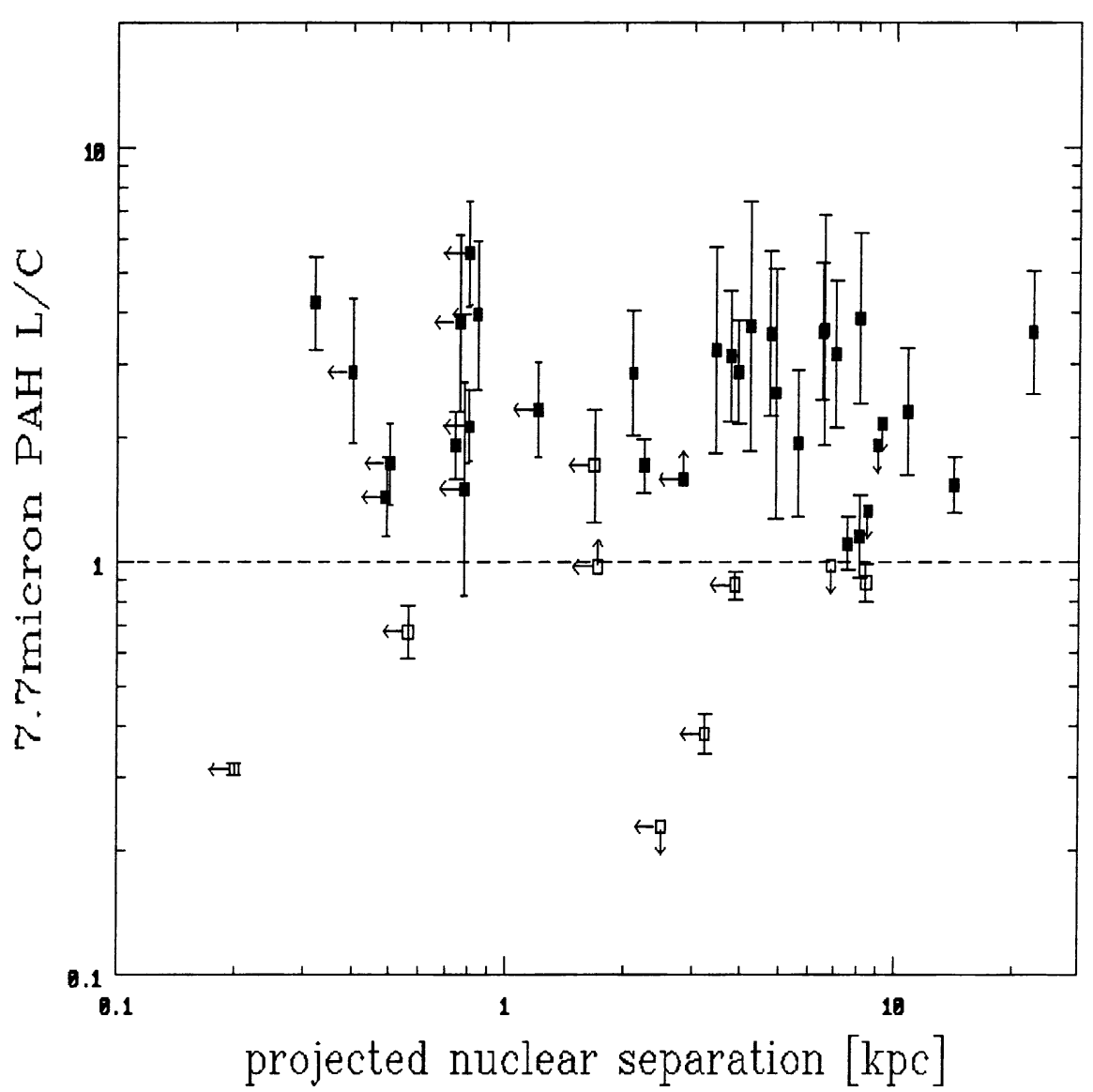

Figure 9. $7.7 \mu \mathrm{m}$ PAH L/C as a function of nuclear separation of the interacting components of a ULIRG (from Rigopoulou et al. 1999). The dashed line indicates the adopted separation between starbursts and $\mathrm{AGN}$ at $\mathrm{L} / \mathrm{C}=1$.

The question then arises whether in these sources the AGNs can be totally hidden even in the mid-infrared. However, in contrast to the hot dust continuum coming from the inner part of the torus, and to the broad line region close to the nucleus, the narrow emission lines of high ionization potential (like [NeVI] at $7.65 \mu \mathrm{m},[\mathrm{NeV}]$ at $14.32 \mu \mathrm{m}, 24.32 \mu \mathrm{m}$ and [OIV] $25.89 \mu \mathrm{m}$ ) are supposed to originate from more extended regions (the narrow line region - NLR) along the ionization cone. High and low excitation lines from the NLR used for probing the hardness of the UV radiation field are hence much less affected by the extinction. The NLR (e.g., [Ne V]) is clearly seen in ISO-SWS spectra of Seyfert 2s (Moorwood et al. 1996; Lutz et al. 2000; Sturm et al. in prep.). In order to completely hide even the narrow line region, dust obscuration must take place 
on much larger spatial scales than a normal torus in normal Seyfert 2s. An alternative explanation would be intrinsically weak or absent narrow line regions in these sources. Seyferts and QSOs with - plausibly intrinsically - weak NLRs are indeed known (e.g., Boroson and Green 1992). For instance, an absorber between BLR and NLR can weaken the NLR. Such an absorber has to be optically thick in the UV, i.e., moderate column densities $\left(10^{19} \mathrm{~cm}^{-3}\right)$ are sufficient (see Alexander et al. 1999 and 2000). These kinds of weak NLR sources would still be classified as QSOs or Seyferts by mid-infrared diagnostics via their warm continuum. The anti-correlation of the submillimeter and X-ray background sources observed so far (e.g., Severgnini et al. 2000; Fabian et al. 2000) argue against a large contribution from totally hidden but dominating AGNs to the ULIRG population. Clearly, a comprehensive archive of highly sensitive midinfrared spectra of a large variety of narrow line regions (in combination with hard X-ray observations) would contribute to solving this puzzle.

Other problems that ISO has left open are, e.g.:

- In starburst-dominated ULIRGs, properties beyond the pure starburst power, for example the excitation, are not well constrained. Also, limits on a possible weak AGN are usually not good enough to test for the frequency of starburst/AGN coexistence.

- The role of metallicity is only partially explored, both in the sense of measuring metallicities in optically inaccessible dusty systems, and in the sense of calibrating infrared spectral properties as a function of metallicity.

- Extended wavelength coverage and higher $\mathrm{S} / \mathrm{N}$ of individual spectra are needed to fully exploit the power of decomposition of low resolution spectra into starburst and AGN components, taking into account extinction. Such data must reliably detect the subtle signatures of dust and ice absorption in order to be useful for making this decomposition.

Future missions, such as SIRTF, SOFIA, or FIRST, will undoubtedly allow us to shed more light on these questions.

\section{References}

Alexander, T., Sturm, E., Lutz, D., et al. 1999, ApJ, 512, 204

Alexander, T., Lutz, D., Sturm, E., et al. 2000, ApJ, 536, 710

Almaini, O., et al. 1998, Astron. Nachr., 319, 55

Barger, A. J., Cowie, L. L., Sanders, D. B., et al. 1999, Nature, 394, 248

Boroson, T. A., \& Green, R. F. 1992, ApJS, 80, 109

Carlberg, R. G., Pritchet, C. J., \& Infante, L. 1994, ApJ, 435, 540

Downes, D., et al. 1999, A\&A, 347, 809

Dwek, E., et al. 1998, ApJ, 508, 106

Fabian, A. C., \& Barcons, X. 1992, ARAA, 30, 429 
Fabian, A. C., et al. 2000, MNRAS, 315, L8

Genzel, R., Lutz, D., \& Tacconi, L. 1998a, Nature, 395, 859

Genzel, R., Lutz, D., Sturm, E., et al. 1998b, ApJ, 498, 579

Genzel, R., \& Cesarsky, C. 2000, ARAA, 38, 761

Guainazzi, M., et al. 2000, A\&A, 356, 463

Hauser, M. G., et al. 1998, ApJ, 508, 25

Heckman, T. 1999, in Proceedings of the Ringberg Workshop, Ultraluminous Galaxies: Monsters or Babies?, ed. D. Lutz \& L. Tacconi, Astrophys. Space Sci., 266, No. 1-2

Holland, W. S., et al. 1999, MNRAS, 303, 659

Hughes, D. H., et al. 1998, Nature, 394, 241

Iwasawa, K., et al. 1993, ApJ, 409, 155

Iwasawa, K., \& Comastri, A. 1998, MNRAS, 297, 1219

Joseph, R. D., \& Wright, G. S. 1985, MNRAS, 214, 87

Joseph, R. D. 1999, Ap\&SS, 266, 321

Kormendy, J., \& Sanders, D. B. 1992, ApJ, 390, L53

Lagache, G., et al. 1999, Ap\&SS, 269, 263

Laurent, O., et al. 1999, Ap\&SS, 269, 357

Laurent, O., et al. 2000, A\&A, 359, 887

Lutz, D., Spoon, H. W. W., Rigopoulou, D. et al. 1998, ApJ, 505, L103

Lutz, D., Veilleux, S., \& Genzel, R. 1999, ApJ, 517, L13

Lutz, D., et al. 2000, ApJ, 530, 733

Madau, P., et al. 1996, MNRAS, 283, 1388

Moorwood, A. F. M., Lutz, D., Oliva, E., et al. 1996, A\&A, 315, L109

Neuschaefer, L. W., et al. 1997, ApJ, 480, 59

Pettini, M., et al. 1998, ApJ, 508, 539

Puget, J.-L., et al. 1996, A\&A, 308, L5

Rigopoulou et al. 1999, AJ, 118, 2625

Sanders D. B., Soifer, B. T., Elias, J. H., et al. 1988, ApJ, 325, 74

Sanders, D. B., \& Mirabel, I. F. 1996, ARAA, 34, 749 
Severgnini, P., et al. 2000, A\&A, 360, 457

Spoon, H. W. W., Koornneef, J., Moorwood, A. F. M., et al. 2000, A\&A, 357, 898

Steidel, C. C., et al. 1999, ApJ, 519, 1

Sturm, E., Lutz, D., Tran, D., et al. 2000, A\&A, 358, 481

Surace, J. A., \& Sanders, D. B. 1999, ApJ, 512, 162

Tran, D., Lutz, D., Genzel, R., et al. 2001, ApJ, in press (astro-ph/0101187)

Zepf, S. E., \& Koo, D. C. 1989, ApJ, 337, 34

\section{Discussion}

Frank Bertoldi: The high-redshift $\mathrm{mm} /$ submm sources are more luminous than the local ULIRGs. Extrapolating the trend you find of starburst-dominated to AGN-dominated for increasing luminosity, these objects should be AGNdominated. To estimate their redshifts from the mm-to-radio flux ratio, however, we assume they are starburst dominated. AGNs tend to show stronger radio emission. Do you find a trend for ULIRGs also to show stronger radio-to-IR flux ratios with increasing IR luminosity? Or with your AGN vs. starburst indicators?

Eckhard Sturm: We have not yet looked into this question.

Jim Peebles: What is known about the redshift-dependence of the ULIRG luminosity function?

Sturm: Most ULIRGs, with the exception of a handful of hyperluminous galaxies, are at redshifts much below $\sim 0.4$, so that we cannot really answer this question, I believe. Hopefully, this will change with the upcoming mid- to far-IR missions, SIRTF, SOFIA, FIRST, ...

Eli Dwek: Have you modeled the strengths of the high excitation and PDR lines in order to probe the physical conditions of the AGN and SB samples?

Sturm: We have used photoionization models in a number of papers. For the starburst sample this is described in Thornley et al. 2000, ApJ, 539, 641. Our detailed studies and modeling of 3 prototypical Seyfert galaxies can be found in Moorwood et al. 1996, A\&A, 315, L109, for the Circinus galaxy; Sturm et al. 1999, ApJ, 512, 197, and Alexander et al. 1999, ApJ, 512, 204, for NGC4151; and Lutz et al. 2000, ApJ, 530, 733 and Alexander et al. 2000, ApJ, 536, 710 for NGC 1068. A paper summarizing the results for the rest of the AGNs observed by us with ISO-SWS is in preparation (Sturm et al. 2001). 